Research Article

\title{
Dynamic Behavior of Externally Prestressed Continuous Beam considering Second-Order Effect
}

\author{
De-Ping Fang \\ College of Civil Engineering, Huaqiao University, Xiamen, China \\ Correspondence should be addressed to De-Ping Fang; fdp@hqu.edu.cn
}

Received 17 June 2020; Revised 5 November 2020; Accepted 10 November 2020; Published 28 December 2020

Academic Editor: Filippo Ubertini

Copyright (C) 2020 De-Ping Fang. This is an open access article distributed under the Creative Commons Attribution License, which permits unrestricted use, distribution, and reproduction in any medium, provided the original work is properly cited.

Considering precisely the second-order deformation of external tendon, the analytical solution of natural frequencies of 2-span externally prestressed continuous beam was obtained by the energy method. The effect of external prestress compression softening is between the zero effect of unbonded prestress compression and the effect of axial outside compression and is determined by the influence coefficient ranging within $0 \sim 1$. The influence coefficient is mainly related to the number of deviators and slightly related to tendon layout. Without deviator, the influence coefficient is 1 , and the effect of external prestress compression softening is the same as the effect of axial outside compression. As the number of deviators increases, the influence coefficient gradually decreases from 1 to near 0 , and the effect of external prestress compression softening is close to zero effect of unbonded prestress compression. With one or more deviators, the effect of external prestress compression softening is negligible. As the eccentricity and area of tendon increase, only the first symmetric frequency increases obviously, and other frequencies almost remain unchanged. The influence of tendon layout linear transformation on the frequency is negligible.

\section{Introduction}

Externally prestressed concrete structure has been widely used in the strengthening of existing concrete bridges as well as in the construction of various new structures. Lou et al. $[1,2]$ and Anwar et al. [3] studied the static nonlinear behavior of beam externally prestressed with steel tendon and FRP cables, and Fang [4] investigated the friction effect of externally prestressed beam. However, the dynamic behavior of externally prestressed beam was not investigated in detail. The analysis of the natural frequencies of externally prestressed simply supported or continuous beams is of vital importance in dynamic behavior. The natural frequency of a simply supported beam under axial outside compression is

$$
\omega_{i}=\frac{i \pi}{l} \sqrt{\frac{1}{m}\left[E I\left(\frac{i \pi}{l}\right)^{2}-N\right]}, \quad i=1,2,3, \ldots,
$$

where $E I$ is flexural stiffness of beam, $m$ is the mass per unit length of beam, $l$ is beam span, $i$ is the mode number, and $N$ is the axial outside compression. Equation (1) reveals that $N$ leads to compression softening ( $N$ softening effect hereinafter) and then decreases the natural frequencies. The prestress tendon applies axial inside compression to the beam. There exists a problem whether the prestress compression softening effect is the same as $N$ softening effect. Different researchers presented different conclusions.

Saiidi et al. [5] determined the natural frequencies of a prestressed concrete bridge using equation (1). Dallasta and Dezi [6] pointed out Saiidi et al.'s [5] approach to consider the prestress compression as $N$ is incorrect, and indicated that the effect of prestress compression on the beam natural frequencies is negligible based on a linear model. Deak [7] also pointed out that prestress compression does not reduce the natural frequencies. However, the view was not supported by any analytical or mathematical proof. Jain and Goel [8] further pointed out that because the tendon becomes an integral part of the system, the prestress compression cannot be treated as $N$ and does not affect the natural frequencies of beams. Jaiswal [9] investigated the first natural frequency of beams by finite element method (FEM). For the beams with bonded tendon, the prestress 
compression does not have any appreciable effect on the first natural frequency. For the beams with unbonded tendon, the first natural frequency significantly changes with the prestress compression and eccentricity of the tendon. Jaiswal's [9] conclusions are only based on FEM results and are not supported by any analytical or mathematical proof. Kanaka [10] treated prestress compression as $N$ and concluded that the prestress compression reduces the natural frequency of the lower modes based on a Rayleigh-Ritz formulation. Chan [11] also treated prestress compression as $N$ and indicated that the natural frequencies of a prestressed bridge decrease as the prestress compression increases. Dallasta and Leoni [12] presented a general formulation for the vibration of concrete beams, prestressed by internal frictionless tendons using kinematic relations of small displacements. The formulation for the beams does not include the effect of the compression; however, the natural frequencies decrease as the prestress compression increases. Kerr [13] studied experimentally and analytically the dynamic response of a prestressed beam and pointed out that the magnitude of the prestress compression for a tendon that passes through the centroid of the beam cross section has zero effect on the dynamic response of the beam. The analytical model was based on a linear formulation to study the dynamic response of the prestressed beam. It was limited to straight tendons that pass through the centroid of the beam. Simsek and Kocaturk [14] indicated that the deflections of the beams increase as the prestress compression increases. The prestress compression softening decreases the beam stiffness and natural frequencies. Wang and Ren [15] considered the additional potential energy of the prestress compression and concluded that the prestress compression reduces the low transverse natural frequencies of the bridge. Jiang and Ye [16] transferred the eccentric unbonded prestress compression to $N$ and a couple and concluded the prestress compression results in compression softening effect. Hamed and Frostig [17] derived the equations of motion for a prestressed beam using the variational principle of virtual work following Hamilton's principle. The mathematical model is rigorous and general and is valid for any kind of boundary and continuity conditions as well as any tendon layout. It was mathematically proven that the prestress compression does not affect the natural frequencies of bonded or unbonded prestressed beams as opposed to some research conclusions.

Hamed and Frostig [17] pointed out zero softening effect of prestress compression of bonded or unbonded tendon. The external tendon applies external prestress compression EP to the beam; does EP lead to softening effect? Miyamoto et al. [18] presented the dynamic behavior of prestressed simply supported beam strengthened with external tendon. EP effect is simply treated as $N$ effect. Shi et al. [19] adopted similar method of Miyamoto et al. [18] to treat EP effect as $N$ effect and obtained the analytical frequency expression of externally prestressed concrete continuous beam. The analytical values were compared with the measured frequencies. Simsek and Kocaturk [20] treated the eccentric EP as $N$ and a couple, and the EP effect is equivalent to $N$ effect. Ji et al. [21] proposed sophisticated formulas to predict the vertical bending vibration frequencies of prestressed concrete box girders with corrugated steel webs based on Hamilton's energy variational principle. The effects of external tendon and EP were not taken into consideration.

There exists the eccentricity loss in external tendon, but there is zero eccentricity loss in unbonded tendon. The loss of external tendon eccentricity decreases the flexural stiffness and consequentially decreases the natural frequencies. The loss of eccentricity depends on the layout of external tendon. Therefore, the influence of EP on the natural frequencies is also related to the layout of external tendon. It can be illustrated simply in Figure 1. During the vibration of a simply supported beam, the internal unbonded tendon $A C B$ along the beam axis bends, but its length and strain energy remain constant. The prestress compression does not influence the dynamic behavior. It conforms with Hamed and Frostig's [17] conclusion of zero effect of prestress compression on the natural frequencies of bonded or unbonded prestressed beams. The external tendon $A B$ anchored at two ends remains straight; therefore it shortens. The loss of tendon $A B$ strain energy transfers to the beam, increases the strain energy and deformation of beam, and decreases the beam stiffness and natural frequency. If $N$ is equal to $E P$, the difference between the loss of $A B$ tendon strain energy and the work of $N$ is high order negligible; $E P$ softening effect is the same as $N$ softening effect and influences the natural frequencies.

Based on energy method, adopting the vibration modes of usual simply supported beam, considering precisely the second-order effect, Fang [22] analyzed the natural frequencies of externally prestressed simply supported beam and EP softening effect. It was concluded that EP softening effect depends on the loss of external tendon eccentricity. As the number of deviators increases from zero to a large number, the layout of the external tendon is close to unbonded tendon. EP softening effect decreases from $N$ effect to almost zero. It is consistent with the conclusion of Hamed and Frostig [17]. Fang [22] only discussed the simply supported beam. The continuous beams were widely adopted in engineering, and the analysis of continuous beam dynamic behavior is also indispensable. Using the method proposed by Fang [22], adopting the vibration modes of usual continuous beam, the natural frequencies of continuous beam were also analyzed, and similar conclusions are presented in this paper.

\section{Natural Frequency Equations of Externally Prestressed Beams}

In the author's view, there exist some problems in Miyamoto et al.'s [18] and Shi et al.'s [19] natural frequency formulas. The derivation procedure is rewritten briefly in this section.

For free vibration, the governing partial differential equation is

$$
\mathrm{EI} \frac{\partial^{4} y}{\partial x^{4}}+\frac{\partial^{2}\left(P_{\mathrm{tc}} y\right)}{\partial x^{2}}-\frac{\partial^{2} \Delta M p}{\partial x^{2}}+m \frac{\partial^{2} y}{\partial t^{2}}=0
$$

where $y$ is vibration displacement; $P_{t}$ and $\Delta P_{t}$ are prestress compression and increment; $P_{t}^{0}$ is initial prestress compression; $P_{\mathrm{tc}}, \Delta P_{\mathrm{tc}}$, and $P_{\mathrm{tc}}^{0}$ are the horizontal components of 


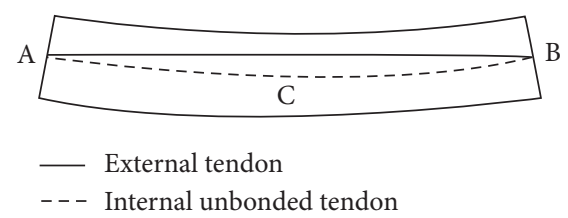

FIGURE 1: Deformation of external and internal unbonded tendons.

$P_{t}, \Delta P_{t}$, and $P_{t}^{0} ; \Delta M_{p}$ is the bending moment due to $\Delta P_{t} ; e$ is the eccentricity; and $a$ is the position of deviators as shown in Figure 2; the friction between the tendon and deviator is neglected. The effect of the prestress compression and the effect of external tendon are represented by the terms $P_{\mathrm{tc}} y$ and $\Delta M_{P}$, respectively, in equation (2)

To solve equation (2), two relationships must be established, the relationship between $P_{\mathrm{tc}} y$ and $y$ and the relationship between $\Delta M_{P}$ and $y$. Miyamoto [18] neglected the term $\Delta P_{\mathrm{tc}} y$ in $P_{\mathrm{tc}} y P_{\mathrm{tc}} y \approx P_{\mathrm{tc}}^{0} y$; since $y_{\max } \ll e$, therefore $\Delta P_{\mathrm{tc}} y \ll \Delta P_{\mathrm{tc}} e\left(\Delta P_{\mathrm{tc}} e\right.$ is included in $\Delta M_{P}$ as shown in Figure 3). In the author's view, $\Delta P_{\text {tc }} y$ is negligibly small, not due to $y_{\max } \ll e$. But it is due to the fact that $\Delta P_{\mathrm{tc}}$ is proportional to $y$, and $y$ can be infinitesimal; $\Delta P_{\text {tc }} y$ becomes a high-order infinitesimal; hence, $\Delta P_{\text {tc }} y$ can be neglected in comparison with infinitesimal $P_{\mathrm{tc}}^{0} y$. Acknowledge that, in some beams, where $e=0, y_{\max } \ll e$ is not a valid assumption. Therefore, $P_{\mathrm{tc}} y$ can be replaced by $P_{\mathrm{tc}}^{0} y$; then, the relationship between $P_{\text {tc }} y$ and $y$ is established. Miyamoto et al. [18] simplified $\Delta M_{P}$ as a uniform bending moment $\Delta M_{\mathrm{PE}}$ as shown in Figure 3 (equivalent-area principle hereinafter):

$$
\Delta M_{P} \approx \Delta M_{\mathrm{PE}}=\Delta P_{t}(e \cos \theta+a \sin \theta) .
$$

Miyamoto [18] assumed that $\Delta P_{t}$ is proportional to midspan displacement $y(0.5 l)$, called proportional assumption hereinafter:

$$
\begin{gathered}
\Delta P_{t}=\eta y(0.5 l), \\
\Delta M_{\mathrm{P}} \approx \Delta M_{\mathrm{PE}}=\eta(e \cos \theta+a \sin \theta) y(0.5 l),
\end{gathered}
$$

where $\quad \eta=24 E I(e \cos \theta+a \sin \theta) / l^{2}(\mu+4 \lambda \cos \theta) \mu=$ $(4 \cos \theta-3)(e \cos \theta+a \sin \theta)^{2} ; \lambda=I / A+E I l_{t} / E_{t} A_{t} l ; A$ is area of the beam cross section; and $E_{t}, A_{t}$, and $l_{t}$ are the elastic modulus, cross section area, and length of tendon. Substituting equation (5) into equation (2) gives

$$
E I \frac{\partial^{4} y}{\partial x^{4}}+P_{\mathrm{tc}}^{0} \frac{\partial^{2}(y)}{\partial x^{2}}-\eta(e \cos \theta+a \sin \theta) \frac{\partial^{2} y(0.5 l)}{\partial x^{2}}+m \frac{\partial^{2} y}{\partial t^{2}}=0,
$$

and $y(0.5 l)$ in equations (4)-(6) is replaced by $y(x)$ in Miyamoto's [18] equations. Due to this replacement, equation (6) can be solved for natural frequencies:

$$
\omega_{i}=\frac{i \pi}{l} \sqrt{\frac{1}{m}\left[\mathrm{EI}\left(\frac{i \pi}{l}\right)^{2}-P_{\mathrm{tc}}^{0}+\eta(e \cos \theta+a \sin \theta)\right]}, \quad i=1,2,3, \ldots
$$

Using a similar method, Shi et al. [19] put forward the natural frequency formula for continuous beam as shown in Figure 4. To solve equation (2), $P_{\mathrm{tc}} y$ is replaced by $P_{\mathrm{tc}}^{0} y$,

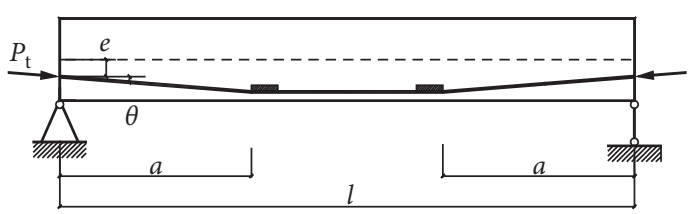

FIGURE 2: Externally prestressed simply supported beam.

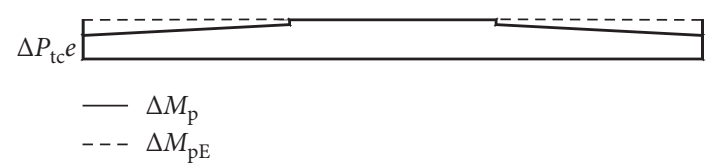

FIgURE 3: Bending moment diagrams $\Delta M_{P}, \Delta M_{\mathrm{PE}}$ of simply supported beam.

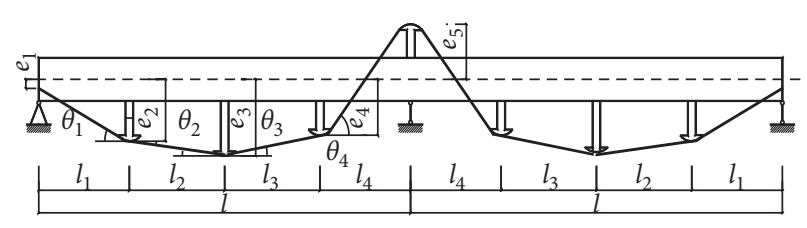

Figure 4: Externally prestressed continuous beam.

$\Delta P_{t}=\phi y(0.5 l)$ by the proportional assumption. Based on the equivalent-area principle, the areas of the bending moment diagram $\Delta M_{P}$ due to $\Delta P_{t}$ are equal to the uniform bending moment $\Delta M_{\mathrm{PE}}$, as shown in Figure $5 \Delta M_{\mathrm{P}}=$ $\Delta M_{\mathrm{PE}}=H \phi y(0.5 l)$. The differential equation similar to equation (6) is obtained:

$$
E I \frac{\partial^{4} y}{\partial x^{4}}+P_{\mathrm{tc}}^{0} \frac{\partial^{2}(y)}{\partial x^{2}}-H \phi \frac{\partial^{2} y(0.5 l)}{\partial x^{2}}+m \frac{\partial^{2} y}{\partial t^{2}}=0 .
$$

Replacing $y(0.5 l)$ by $y(x)$, solving equation (8) for natural frequencies of continuous beam, the expressions of $H, \phi$ and frequencies are detailed in the paper by Shi et al. [19].

\section{Some Problems in Proportional Assumption and Equivalent-Area Principle}

In the derivation of Miyamoto et al.'s [18] and Shi et al.'s [19] analytical expressions of the natural frequencies, in the author's view, there are 4 problems in the proportional assumption and equivalent-area principle.

(1) In proportional assumption, the increment of tendon tension $\Delta P_{t}$ is proportional to the vibration displacement at midspan of beam $y(0.5 l)$; the proportional coefficient is a constant in equation (4). But for different vibration mode, the same $y(0.5 l)$ results in different deformation and $\Delta P_{t}$ of tendon, the proportional assumption is not valid.

(2) In order to solve equations (6) and (8) conveniently, $y(0.5 l)$ is replaced by $y(x)$; i.e., $\Delta P_{t}$ is not proportional to $y(0.5 l)$ but is proportional to $y(x)$. This replacement is wrong because $\Delta P_{t}$ is constant along 


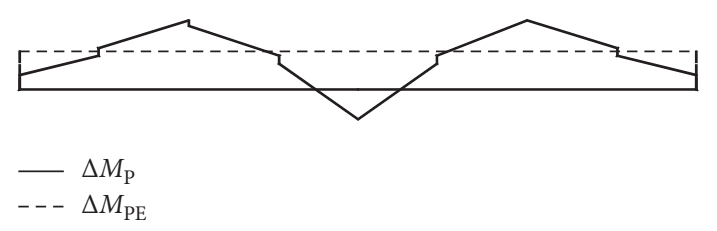

Figure 5: Bending moment diagrams $\Delta M_{\mathrm{P}}, \Delta M_{\mathrm{PE}}$ of continuous beam.

the whole tendon without friction, but the value $y(x)$ varies along $x$.

(3) Also for solving convenience, the equivalent-area principle simplifies $\Delta M_{\mathrm{P}}$ into uniform $\Delta M_{\mathrm{PE}}$, which results in negligible error in simply supported beam for small $a$ in Figure 2, but it would result in obvious error in continuous beam. In Figure 5, both midspan bending moment above axis and bending moment below axis near the middle support contribute to stiffness of continuous beam in spite of plus-minus sign. The calculation of $\Delta M_{\mathrm{PE}}$ is the area of $\Delta M_{\mathrm{P}}$ above axis minus the area of $\Delta M_{\mathrm{P}}$ below axis; two bending moments originally strengthen together and now wrongly counteract mutually. The continuous beam deflects upward under uniform $\Delta M_{\mathrm{PE}}$, which does not meet zero displacement at middle support, so obvious error is expected in the equivalent-area principle.

(4) $P_{\mathrm{tc}}^{0} y$ in equations (6) and (8) implies EP effect is treated as $N$ effect without considering the influence of deviators, which is not consistent with Fang [22] conclusion.

Therefore, it is imperative to develop more accurate analytical solution for continuous beam frequencies considering the second-order effects without the above problems.

\section{Energy Method to Analyze the Natural Frequencies of Continuous Beam}

In the present study, the energy method considering the second-order effects is adopted to solve the problems that existed in Shi et al.'s [19] solution, and to find the natural frequencies of the continuous beam shown in Figure 4. The assumptions in the energy method are as follows:

(1) The ith vibration mode $y_{i}=A_{i} X_{i}(x) \sin \left(\omega_{i} t\right) ; A_{i}$ : amplitude of $i$ th mode; antisymmetric $i$ th mode: $X_{i}$ $(x)=\sin \left(k_{i} x\right), k_{i} l=i \pi, i=1,2,3 \ldots$ symmetric $i$ thmode: $X_{i}(x)=\sin \left(k_{i} x\right)-f_{i} \sinh \left(k_{i} x\right), f_{i}=\sin \left(k_{i} l\right) / \sinh \left(k_{i} l\right)$, $k_{i} l=(i+0.25) \pi, i=1,2,3, \ldots$ both symmetric and antisymmetric modes are the same as modes of usual continuous beam.

(2) The beam is straight after applying prestress and before vibrating.
(3) The axial deformation of the beam due to axial compression and the mass of tendon and deviators are neglected.

When $\dot{y}_{i}=\max$ or $y_{i}=0$, the strain energy of the beam is

$$
U_{b 0}=\frac{1}{2 E I} \int_{0}^{2 l}\left(P_{\mathrm{t}}^{0} m_{t}\right)^{2} \mathrm{~d} x,
$$

where $P_{\mathrm{t}}^{0} m_{t}$ is the bending moment due to $P_{\mathrm{t}}^{0}$ and $m_{t}$ is the bending moment due to the unit tension of external tendon; $m_{t}=\Delta M_{\mathrm{p}} / \Delta P_{t}$ in Figure 5.

The kinetic energy of the beam is

$$
K_{b 0}=\frac{1}{2} \int_{0}^{2 l} m \dot{y}_{i}^{2} d x=A_{i}^{2} \omega_{i}^{2} r_{b 0} m .
$$

For antisymmetric $i$ th mode, $r_{b 0}=l / 2$; for symmetric $i$ th, mode, $r_{b 0}=l / 2-1 / 4 k_{i}+f_{i}^{2}\left[\sinh \left(2 k_{i} l\right)-2 k_{i} l\right] / 4 k_{i}-f_{i}[\sin$ $\left.\left(k_{i} l\right) \cosh \left(k_{i} l\right)-\cos \left(k_{i} l\right) \sinh \left(k_{i} l\right)\right] / k_{i}$. The strain energy of external tendon is

$$
U_{t 0}=\frac{\left(P_{t}^{0}\right)^{2} l_{t}}{2 E_{t} A_{t}}
$$

When $y_{i}=\max$ or $\dot{y}_{i}=0$, the bending moment of beam is $M_{\mathrm{b} 1}=-\mathrm{EI} y_{i}^{\prime \prime}-P_{\mathrm{t}}^{0} m_{t}$, and the strain energy of the beam is

$$
U_{b 1}=\frac{1}{2 E I} \int_{0}^{2 l} M_{b 1}^{2} d x=\operatorname{EIA}_{i}^{2} r_{b 1}+\int_{0}^{2 l} y_{i}^{\prime \prime} P_{t}^{0} m_{t} \mathrm{~d} x+U_{b 0} .
$$

For antisymmetric ith mode, $r_{b 1}=k_{i}^{4} l / 2$; for symmetric $i$ th mode, $r_{b 1}=k_{i}^{3}\left\{k_{i} l / 2-1 / 4+f_{i}^{2} \quad\left[\sinh \left(2 k_{i} l\right)-2 k_{i} l\right] /\right.$ $\left.4+f_{i}\left[\sin \left(k_{i} l\right) \cosh \left(k_{i} l\right)-\cos \left(k_{i} l\right) \sinh \left(k_{i} l\right)\right]\right\}$.

As for calculating the deformation of the external tendon, the deformation of line OA in Figure 6 is investigated firstly. The deformations of line OA in $x$ and $y$ axes are $\delta x$ and $\delta y$; considering the second differentiation, the deformation of line $\mathrm{OA}$ is

$$
\begin{aligned}
O \bar{A}-O A= & \sin \theta \delta y+\cos \theta \delta x+\frac{(\sin \theta \delta x)^{2}}{2 l_{A}} \\
& +\frac{(\cos \theta \delta y)^{2}}{2 l_{A}}-\frac{\sin \theta \cos \theta \delta x \delta y}{l_{A}},
\end{aligned}
$$

where $\theta$ and $l_{A}$ are angle and length of line OA. It is indispensable to consider the second differentiation in analyzing $E P$ softening. There are $n$ contact points between the beam and external tendon, the first and the last contact points are two anchorages at two ends of the beam, and the remaining others are at the deviators. In the $j$ th segment of external tendon as shown in Figure 7, the coordinates of the $j$ th and $(j+1)$ th contact points are $\left(x_{j}, e_{j}\right)$ and $\left(x_{j+1}, e_{j+1}\right)$ before vibrating. Considering the horizontal displacement of the point at beam axis due to the axis bending, the coordinates of the contact points $\left(\bar{x}_{j}, \bar{y}_{j}\right)$ and $\left(\bar{x}_{j+1}, \bar{y}_{j+1}\right)$ when $\dot{y}_{i}=0$ are 


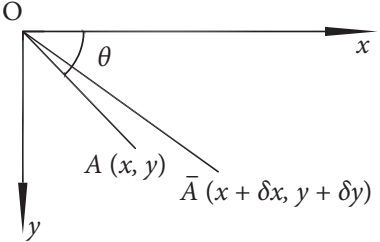

Figure 6: Deformation of line OA.

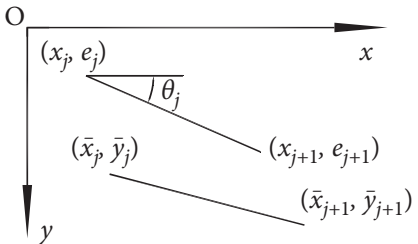

Figure 7: Deformation of external tendon segment.

$$
\begin{aligned}
\bar{x}_{j} & =x_{j}-\frac{1}{2} \int_{0}^{x_{j}}\left(y_{i}^{\prime}\right)^{2} \mathrm{~d} x-e_{j} y_{i}^{\prime}\left(x_{j}\right), \\
\bar{x}_{j+1} & =x_{j+1}-\frac{1}{2} \int_{0}^{x_{j+1}}\left(y_{i}^{\prime}\right)^{2} \mathrm{~d} x-e_{j+1} y_{i}^{\prime}\left(x_{j+1}\right), \\
\bar{y}_{j} & =e_{j}+y_{i}\left(x_{j}\right), \\
\bar{y}_{j+1} & =e_{j+1}+y_{i}\left(x_{j+1}\right) .
\end{aligned}
$$

The deformations of the $j$ th segment in $x$ and $y$ axes $\delta x_{j}$ and $\delta y_{j}$ are

$$
\begin{aligned}
\delta x_{j} & =\left(\bar{x}_{j+1}-\bar{x}_{j}\right)-\left(x_{j+1}-x_{j}\right)=b_{j} A_{i}^{2}+c_{j} A_{i}, \\
\delta y_{j} & =\left(\bar{y}_{j+1}-\bar{y}_{j}\right)-\left(e_{j+1}-e_{j}\right)=d_{j} A_{i}, \\
b_{j} & =f\left(x_{j}\right)-f\left(x_{j+1}\right), \\
c_{j} & =e_{j} X_{i}^{\prime}\left(x_{j}\right)-e_{j+1} X_{i}^{\prime}\left(x_{j+1}\right), \\
d_{j} & =X_{i}\left(x_{j+1}\right)-X_{i}\left(x_{j}\right), \\
f(x) & =\frac{1}{2} \int_{0}^{x}\left(X_{i}^{\prime}\right)^{2} \mathrm{~d} x .
\end{aligned}
$$

For antisymmetric $i$ th mode, $f(x)=0.125 k_{i}\left[2 k_{i} x+\sin \right.$ $\left.\left(2 k_{i} x\right)\right]$; for symmetric $i$ th, mode $f(x)=0.125 k_{i}\left\{2 k_{i} x+\right.$ $\sin \left(2 k_{i} x\right)+f_{i}^{2}\left[\sinh \left(2 k_{i} x\right)+2 k_{i} x\right]-4 f_{i}\left[\sinh \left(k_{i} x\right) \cos \left(k_{i} x\right)\right.$ $\left.\left.+\cosh \left(k_{i} x\right) \sin \left(k_{i} x\right)\right]\right\}$. Substituting $\delta x_{j}$ and $\delta y_{j}$ into equation (13), and neglecting the terms involving high-order infinitesimal $A_{i}^{3}$ and $A_{i}^{4}$ ( $A_{i}$ can be infinitesimal), give the deformation of the $j$ th segment of external tendon:

$$
\Delta l_{j}=\alpha_{j} A_{i}+\beta_{j} A_{i}^{2}
$$

$\alpha_{j}=d_{j} \sin \theta_{j}+c_{j} \cos \theta_{j}, \quad \beta_{j}=b_{j} \cos \theta_{j}+\left(c_{j} \sin \theta_{j}\right)^{2} / 2 l_{j}+$ $\left(d_{j} \cos \theta_{j}\right)^{2} / 2 l_{j}-c_{j} d_{j} \sin \theta_{j} \cos \theta_{j} / l_{j} ; \theta_{j}$ and $l_{j}$ are the length and angle of the $j$ th segment of external tendon, respectively. The whole deformation of the external tendon is

$$
\Delta l_{t}=A_{i} \psi_{i}+A_{i}^{2} \zeta_{i},
$$

where $\psi_{i}=\sum_{j=1}^{n-1} \alpha_{j}, \zeta_{i}=\sum_{j=1}^{n-1} \beta_{j} A_{i} \psi_{i}$ is the first-order deformation due to the $i$ th vibration mode; i.e., $A_{i} \psi_{i}=-\int_{0}^{2 l} y_{i}^{\prime \prime} m_{t} \mathrm{~d} x$. For antisymmetric $i$ th mode, $A_{i} \psi_{i}=0$. $A_{i}^{2} \zeta_{i}$ is the second-order deformation, which involves not only the horizontal projection variation of contact point due to the axis bending, i.e., $b_{j}$ term, but also $c_{j}^{2}, d_{j}^{2}, c_{j} d_{j}$ terms in equation (16). If considering only $b_{j}$ term, it results in incorrect conclusion: EP effect is the same as $N$ effect. Precisely considering $c_{j}^{2}, d_{j}^{2}, c_{j} d_{j}$ terms leads to correct conclusion: EP effect is between $N$ effect and zero effect of unbonded prestress compression. The tension of external tendon is

$$
P_{t 1}=P_{t}^{0}+\frac{E_{t} A_{t}\left(A_{i} \psi_{i}+A_{i}^{2} \zeta_{i}\right)}{l_{t}} .
$$

The strain energy of external tendon is $U_{t 1}=P_{t 1}^{2} l_{t} / 2 E_{t} A_{t}$; neglecting the terms involving high-order infinitesimal $A_{i}^{3}$ and $A_{i}^{4}$ gives

$$
U_{t 1}=U_{t 0}+P_{t}^{0}\left(A_{i} \psi_{i}+A_{i}^{2} \zeta_{i}\right)+\frac{E_{t} A_{t}\left(A_{i} \psi_{i}\right)^{2}}{2 l_{t}} .
$$

Substituting each term in equations (9)-(12) and (19) into the equation of energy method, $U_{\mathrm{b} 0}+K_{\mathrm{b} 0}+U_{\mathrm{t} 0}=$ $U_{\mathrm{b} 1}+U_{\mathrm{t} 1}$, and deducting the term $\int_{0}^{2 l} y_{i}^{\prime \prime} P_{t}^{0} m_{t} \mathrm{~d} x$ in $U_{\mathrm{b} 1}$ and the term $P_{t}^{0} A_{i} \psi_{i}=-P_{t}^{0} \int_{0}^{2 l} y_{i}^{\prime \prime} m_{t} \mathrm{~d} x$ in $U_{\mathrm{t} 1}$ mutually give

$$
A_{i}^{2} \omega_{i}^{2} r_{\mathrm{b} 0} m=\mathrm{EI} A_{i}^{2} r_{\mathrm{b} 1}+P_{\mathrm{t}}^{0} A_{i}^{2} \zeta_{i}+\frac{E_{t} A_{t}\left(A_{i} \psi_{i}\right)^{2}}{2 l_{t}} \text {. }
$$

The natural frequency is $\omega_{i}=$ $\sqrt{1 / r_{\mathrm{b} 0} m\left[\mathrm{EI} r_{\mathrm{b} 1}+P_{\mathrm{t}}^{0} \zeta_{i}+E_{t} A_{t} \psi_{i}^{2} / 2 l_{t}\right]}$. For antisymmetric $i$ th mode, $\psi_{i}=0 ; \omega_{i}$ can be simplified as

$$
\omega_{i}=\frac{i \pi}{l} \sqrt{\frac{1}{m}\left[\operatorname{EI}\left(\frac{i \pi}{l}\right)^{2}-C_{p i} P_{t}^{0}\right]}, \quad i=1,2,3 \ldots
$$

The influence coefficient $C_{\mathrm{p} i}=-2 l \zeta_{i} /(i \pi)^{2} 0<C_{\mathrm{p} i} \leq 1$; it reflects $E P$ softening effect. For symmetric $i$ th mode, there is no concise analytical frequency solution of usual continuous beam under N. $C_{\mathrm{p} i}$ is defined as follows: when $P_{\mathrm{t}}^{0}=0, y_{i}=$ $\max$ under $N$; the beam axial horizontal length shortens $2 A_{i}^{2} f(l)$ due to axis bending; the equation of energy method: $K_{\mathrm{b} 0}=U_{\mathrm{t} 1}+U_{\mathrm{b} 1}-2 A_{i}^{2} f(l) N, \quad$ obtaining $\quad \omega_{i}=$ $\sqrt{1 / r_{b 0} m\left[E I r_{b 1}-N 2 f(l)+E_{t} A_{t} \psi_{i}^{2} / 2 l_{t}\right]}$; when $P_{t}^{0}=N$, the influence coefficient $C_{p i}=-\zeta_{i} / 2 f(l) \omega_{i}$ for the symmetric mode is

$$
\omega_{i}=\sqrt{\frac{1}{r_{b 0} m}\left[E I r_{b 1}-C_{p i} P_{t}^{0} 2 f(l)+\frac{E_{t} A_{t} \psi_{i}^{2}}{2 l_{t}}\right]}, \quad i=1,2,3 \ldots
$$

In comparison of equations (21) and (22) with equation (1) under $N, E P$ or $P_{t}^{0}$ does affect the natural frequencies of beam, but its effect depends on the coefficient $C_{p i}$ which is related to the layout of external tendon. When $C_{p i}=1, \mathrm{EP}$ 
effect is the same as $N$ effect, similar to the straight external tendon $\mathrm{AB}$ in Figure 1 when $C_{p i}=0$, zero EP softening effect, similar to the internal unbonded tendon $A C B$ in Figure 1.

\section{Numerical Examples}

For the externally prestressed continuous concrete beam shown in Figure $4, l=5 \mathrm{~m}$, with width $0.4 \mathrm{~m}$ and height $0.15 \mathrm{~m}$ of rectangular cross section; $E=32.5 \mathrm{GPa}$; the mass per unit length of the beam $m=0.8 \mathrm{t} / \mathrm{m}$; external tendon $E_{\mathrm{t}}=200 \mathrm{GPa}$; the initial prestress $\sigma_{\mathrm{t}}^{0}=1000 \mathrm{MPa}$; $l_{1}=l_{2}=l_{3}=l_{4}=1.25 \mathrm{~m}$; and the deviators are distributed uniformly spaced along the span. 8 beams were studied with 2 layouts of tendon $(A, B), 2$ two eccentricities (large eccentricity $L$, small eccentricity $S$ ), and two areas of the tendon ( 1 tendon $A_{t 1}=137 \mathrm{~mm}^{2}, 2$ tendons $A_{t 2}=274 \mathrm{~mm}^{2}$ ), which are listed in Table 1 . For all layouts of tendon, $e_{1}=0$ (see Figure 4); all deviators are located at parabola. A-layout can be linearly transformed into B-layout. Large eccentricity $L$ is twice as large as small eccentricity $\mathrm{S}$.

Table 2 shows the relationship between influence coefficient $C_{\mathrm{p} i}$ and the number of contact points $n$ for straight tendon, the straight tendon is along the axis of beam, and the contact points are uniformly distributed. Table 3 shows the relationship between influence coefficient $C_{\mathrm{p} i}$ and the number of contact points $n$ for parabolic tendon; the contact points are also uniformly distributed and located at the same parabola of AL tendon. The results of other parabolic tendon layouts (AS, BL, BS) are almost the same as the results of AL tendon. It is found that the influence coefficient $C_{\mathrm{p} i}$ is mainly related to the contact points $n$ and slightly related to tendon layout. Table 2 indicates that, for the beam with only 3 contact points, i.e., without deviator along span, $C_{p}=1, E P$ effect is the same as $N$ effect, because the loss of eccentricity is maximum, similar to the straight external tendon $\mathrm{AB}$ in Figure 1. As the number of contact points increases, the loss of eccentricityreduces, and the external tendon is gradually close to the unbonded tendon. The influence coefficient decreases from 1 to almost 0 , and EP softening effect also decreases to almost 0 . It conforms with the conclusion pointed out by Hamed and Frostig [17], i.e., zero effect of prestress compression of bonded or unbonded tendon on the natural frequencies. As the number of vibration modes increases, the distance between points of inflection $\left(y^{\prime}=0\right)$ shortens, and the number of contact points between two inflection points decreases, which is equivalent to the reduction of contact points in the first antisymmetric mode; hence, the influence coefficient increases.

M(1), M(2), and M(3) represent the methods in this paper, Shi et al. [19], and FEM, respectively. Generally speaking, the validity of theoretical results would be tested by experimental results. However, in the field and laboratory experiments, Saiidi et al. [5] found that the natural frequencies increase as the prestress compression increases, which is not consistent with the theoretical conclusion. For this disparity, they opined that the prestress compression causes closure of microcracks in the concrete, which increases the flexural stiffness and natural frequencies. In the
TABLE 1: Layouts and eccentricities of external tendons.

\begin{tabular}{lcccc}
\hline Beam code & $e_{2}(\mathrm{~mm})$ & $e_{3}(\mathrm{~mm})$ & $e_{4}(\mathrm{~mm})$ & $e_{5}(\mathrm{~mm})$ \\
\hline AL & 406 & 500 & 281 & 250 \\
AS & 203 & 250 & 141 & 125 \\
BL & 344 & 375 & 94 & 500 \\
BS & 172 & 188 & 47 & 250 \\
\hline
\end{tabular}

TABLe 2: Relationship between influence coefficient $C_{\mathrm{p} i}$ and the number of contact points $n$ for straight tendon.

\begin{tabular}{lcccccc}
\hline & \multicolumn{3}{c}{ Antisymmetric mode } & \multicolumn{3}{c}{ Symmetric mode } \\
& $C_{\mathrm{p} 1}$ & $C_{\mathrm{p} 2}$ & $C_{\mathrm{p} 3}$ & $C_{\mathrm{p} 1}$ & $C_{\mathrm{p} 2}$ & $C_{\mathrm{p} 3}$ \\
\hline 3 & 1 & 1 & 1 & 1 & 1 & 1 \\
5 & 0.189 & 1 & 0.91 & 0.275 & 0.97 & 0.928 \\
7 & 0.088 & 0.316 & 1 & 0.161 & 0.335 & 0.939 \\
9 & 0.05 & 0.189 & 0.385 & 0.098 & 0.241 & 0.372 \\
11 & 0.033 & 0.125 & 0.263 & 0.065 & 0.169 & 0.296 \\
13 & 0.023 & 0.088 & 0.189 & 0.046 & 0.122 & 0.225 \\
17 & 0.013 & 0.05 & 0.11 & 0.026 & 0.072 & 0.137 \\
21 & 0.008 & 0.033 & 0.072 & 0.017 & 0.047 & 0.091 \\
\hline
\end{tabular}

TABle 3: Relationship between influence coefficient $C_{\mathrm{p} i}$ and the number of contact points $n$ for AL tendon.

\begin{tabular}{lcccccc}
\hline & \multicolumn{3}{c}{ Antisymmetric mode } & \multicolumn{3}{c}{ Symmetric mode } \\
& $C_{\mathrm{p} 1}$ & $C_{\mathrm{p} 2}$ & $C_{\mathrm{p} 3}$ & $C_{\mathrm{p} 1}$ & $C_{\mathrm{p} 2}$ & $C_{\mathrm{p} 3}$ \\
\hline 5 & 0.197 & 0.898 & 0.967 & 0.304 & 0.901 & 0.944 \\
7 & 0.036 & 0.335 & 0.96 & 0.15 & 0.362 & 0.921 \\
9 & 0.025 & 0.127 & 0.428 & 0.078 & 0.214 & 0.412 \\
11 & 0.012 & 0.045 & 0.208 & 0.037 & 0.135 & 0.271 \\
13 & 0.01 & 0.02 & 0.113 & 0.013 & 0.081 & 0.195 \\
17 & 0.006 & 0.011 & 0.018 & 0.01 & 0.019 & 0.093 \\
21 & 0.003 & 0.006 & 0.01 & 0.005 & 0.01 & 0.037 \\
\hline
\end{tabular}

experiment, the frictionless slip does not occur; even friction slip hardly occurs due to tight compression between deviator and external tendon under small vibration amplitude, so the experimental results may not validate the theoretical results. For this reason, the theoretical results are validated by FEM results in the present study.

There exists the frictionless slip between the external tendon and deviator; the tension in external tendon is constant along whole length and is decided by whole beam deformation, which results in an additional difficult in FEM. Fang [22] proposed a simple method to solve this difficulty by adding a contact link element at angle bisector between external tendon and deviator as shown in Figure 8. The angle bisector position ensures the uniform increment of tension of external tendon, so the contact link element can simulate the frictionless slip. The length of contact link element is $1 \mathrm{~mm}$, small enough to maintain the tendon layout unchanged; its axial stiffness is $E_{t} A_{t}$. The external tendon is subjected to only axial tension and is simulated by the link element. The deviator is simulated by the rigid beam element. The lumped mass method is adopted at two ends of the beam element. The beam is discretized into 16 beam elements with the same length $0.625 \mathrm{~m}$, each lumped mass $0.5 \mathrm{t}$. The equation to calculate the natural frequencies is 


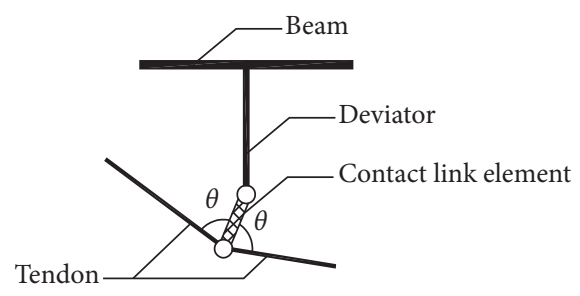

FIGURE 8: Contact link element.

$$
\omega_{i}^{2}[\delta][M]\left\{\varphi_{i}\right\}=\left\{\varphi_{i}\right\}
$$

where $\left\{\varphi_{i}\right\}$ is the $i$ th natural mode, $[M]$ is the diagonal lumped mass matrix, and $[\delta]$ is the vertical flexibility matrix. The influence coefficients of $C_{\mathrm{p} i}$ and $P_{\mathrm{t}}^{0}$ in equations (21) and (22) are taken into consideration; hence, the stiffness matrix of beam element is

$$
\begin{array}{r}
{[K]=\frac{E I}{l^{3}}\left[\begin{array}{cccc}
12 & 6 l & -12 & 6 l \\
6 l & 4 l^{2} & -6 l & 2 l^{2} \\
-12 & -6 l & 12 & -6 l \\
6 l & 2 l^{2} & -6 l & 4 l^{2}
\end{array}\right]} \\
-\frac{C_{\mathrm{p} i} P_{\mathrm{t}}^{0}}{30 l}\left[\begin{array}{cccc}
36 & 3 l & -36 & 3 l \\
3 l & 4 l^{2} & -3 l & -l^{2} \\
-36 & -3 l & 36 & -3 l \\
3 l & -l^{2} & -3 l & 4 l^{2}
\end{array}\right] .
\end{array}
$$

$C_{\mathrm{p} i} P_{\mathrm{t}}^{0}$ reduces the stiffness of beam element, lowers the frequency, and reflects $E P$ softening. $[\delta]$ is computed by standard procedures of structural analysis and involves various parameters, such as $E I, l, C_{\mathrm{p} i}$ in beam stiffness matrix $E_{t} A_{t}, l_{t}$ and the layout of external tendon, the frictionless slip simulated by contact link element, etc. $\omega_{1} \sim \omega_{14}$ are determined by MATLAB software for 14 lumped masses. The lumped mass method provides high accuracy in calculating low-order natural frequencies, so the high accuracy of $\omega_{1} \sim \omega_{4}$ for 8 beams AS1 BL2 listed in Table 4 is expected. Hence, the results of M(3) in Table 4 can be used to validate the accuracy of the results of M(1) and M(2).

The first and second frequencies of antisymmetric modes are $\omega_{1}, \omega_{3}$; the first and second frequencies of symmetric modes are $\omega_{2}, \omega_{4}$; in Table $4, \omega_{1} \sim \omega_{4}$ values of M(1) are very close to the values of M(3), indicating the accuracy of M(1) and M(3). In M(2) method, the equivalent-area principle as shown in Figure 5 wrongly assumes the mutually strengthening effect of bending moment above axis and bending moment below axis on beam stiffness as mutually counteracting effect, which significantly underestimates the effect of external tendon. The equivalent-area principle approximately neglects the effect of external tendon for all frequencies. $\omega_{i}$ of M(2) is slightly less than $\omega_{i}$ of beam 0 without tendon in Table 5, the compression softening effect of $P_{\mathrm{t}}^{0}$ on frequencies is small, and the effect of external tendon is significantly underestimated. The error in M(2) method is obvious. In M(1) method, for symmetric mode $\psi_{1} \gg \psi_{i} \approx 0, i \geq 2$ in equation (17). The first-order tendon deformation is caused almost only by the first symmetric mode, very little by other symmetric modes; therefore, the stiffness of tendon $E_{t} A_{t}$ mainly increases the frequency of the first symmetric mode $\left(\omega_{2}\right)$ and slightly influences the frequency of the other symmetric modes $\left(\omega_{4}, \omega_{6} \ldots\right)$ in equation (22). The stiffness of tendon $E_{t} A_{t}$ does not influence the frequencies of the antisymmetric modes $\left(\omega_{1}\right.$, $\left.\omega_{3} \ldots\right)$ in equation (21). Comparing $\omega_{2}$ values of M(1) among 8 beams, it shows that as the eccentricity and tendon area increase, $\omega_{2}$ values increase, but $\omega_{2}$ values are slightly related to the tendon A or B layout, so the influence of tendon layout linear transformation on the frequency is negligible. In M(1) method, the effect of external tendon on $\omega_{1}, \omega_{3}$ is zero, and the effect of external tendon on $\omega_{4}$ is negligible. In M(2) method, the effect of external tendon on all $\omega_{i}$ is negligible due to the equivalent-area principle. Comparing results of M(1) and M(2), it is found that $\omega_{1}, \omega_{3}$, and $\omega_{4}$ of M(1) are slightly greater than the results of M(2), because M(2) method wrongly treats EP effect as $N$ effect in equation (8); i.e., $C_{\mathrm{p} i}=1 ; \omega_{2}$ of M(1) is obviously greater than $\omega_{2}$ of M(2), because M(1) considers the effect of external tendon $\psi_{1}$ in equation (21); M(2) method still neglects the effect of external tendon due to the equivalent-area principle.

Due to zero effect of external tendon on $\omega_{1}, \omega_{3}$ and no prestress compression $P_{\mathrm{t}}^{0}=0, \omega_{1}, \omega_{3}$ for every beam are the same in Table 5 . The frequencies of 8 beams AS1 BL2 without prestress in Table 5 are quite close to M(1) frequencies in Table 4 with the prestress, indicating EP effect is negligible. In equation. (21), $C_{\mathrm{p} 1}$ is much less than 1 for one or more deviators ( $n \geq 5$ in Tables 2 and 3$)$ along beam span; usually $P_{\mathrm{t}}^{0} \ll E I(\pi / l)^{2}$ the critical load of stability, $E P$ effect, or $C_{\mathrm{p} 1} P_{\mathrm{t}}^{0}$ on $\omega_{1}$ is slight. For high-order frequencies $\omega_{i}, i \geq 2$, $C_{\mathrm{p} i}$ values increase, but the high-order critical loads of stability $E I(i \pi / l)^{2}$ increase more rapidly, and EP effect on high-order frequency is also slight. Equation (22) also presents the same conclusion. In a word, EP effect on all order frequencies is negligible for the beam with one or more deviators. Comparing $\omega_{1} \sim \omega_{4}$ in M(1) Table 4 and in beam 0 Table 5 , it is found that the effect of external tendon on $\omega_{2}$ is obvious, and the effect of external tendon on other frequencies is slight and negligible. 
TABLE 4: The $1^{\text {st }} \sim 4^{\text {th }}$ natural frequency calculation results of externally prestressed continuous beam.

\begin{tabular}{|c|c|c|c|c|c|c|c|c|c|c|c|c|}
\hline \multirow{2}{*}{ Beam code } & \multicolumn{4}{|c|}{ M(1) (rad/s) } & \multicolumn{4}{|c|}{ M(2) (rad/s) } & \multicolumn{4}{|c|}{ M(3) (rad/s) } \\
\hline & $\omega_{1}$ & $\omega_{2}$ & $\omega_{3}$ & $\omega_{4}$ & $\omega_{1}$ & $\omega_{2}$ & $\omega_{3}$ & $\omega_{4}$ & $\omega_{1}$ & $\omega_{2}$ & $\omega_{3}$ & $\omega_{4}$ \\
\hline AS1 & 26.65 & 44.31 & 106.54 & 134.88 & 25.58 & 40.87 & 105.67 & 134.18 & 26.64 & 44.26 & 106.5 & 134.8 \\
\hline AS2 & 26.61 & 46.77 & 106.31 & 134.64 & 24.37 & 40 & 104.51 & 133.93 & 26.6 & 46.6 & 106.28 & 134.57 \\
\hline AL1 & 26.66 & 51.07 & 106.6 & 134.95 & 25.89 & 41.11 & 105.97 & 134.44 & 26.67 & 50.92 & 106.56 & 134.88 \\
\hline AL2 & 26.63 & 58.97 & 106.43 & 134.8 & 24.78 & 40.29 & 104.9 & 133.52 & 26.64 & 58.49 & 106.4 & 134.74 \\
\hline $\mathrm{BS} 1$ & 26.63 & 44.28 & 106.55 & 134.87 & 25.58 & 40.87 & 105.66 & 134.18 & 26.64 & 44.22 & 106.51 & 134.79 \\
\hline BS2 & 26.58 & 46.7 & 106.34 & 134.62 & 24.37 & 40 & 104.51 & 133.19 & 26.57 & 46.55 & 106.32 & 134.55 \\
\hline BL1 & 26.62 & 50.71 & 106.58 & 134.88 & 25.89 & 41.1 & 105.96 & 134.44 & 26.61 & 50.56 & 106.55 & 134.8 \\
\hline BL2 & 26.56 & 58.34 & 106.4 & 134.65 & 24.77 & 40.29 & 104.89 & 133.52 & 26.57 & 58.19 & 106.37 & 134.57 \\
\hline
\end{tabular}

Note: M(1) M(2), and M(3) represent the methods of this paper, Shi et al. [19], and FEM.

TABLE 5: $\omega_{2}, \omega_{4}$ of beams without prestress in M(1).

\begin{tabular}{lccccccccc}
\hline Beam & 0 & AS1 & AS2 & AL1 & AL2 & BS1 & BS2 & BL1 & BL2 \\
\hline$\omega_{2}(\mathrm{rad} / \mathrm{s})$ & 41.7 & 44.39 & 46.92 & 51.13 & 59.07 & 44.36 & 46.86 & 50.79 & 58.48 \\
$\omega_{4}(\mathrm{rad} / \mathrm{s})$ & 135.11 & 135.13 & 135.16 & 135.19 & 135.26 & 135.13 & 135.15 & 135.14 & 135.18 \\
\hline
\end{tabular}

Note: beam 0 denotes beam without external tendon; $\omega_{1}=26.69 \mathrm{rad} / \mathrm{s}, \omega_{3}=106.76 \mathrm{rad} / \mathrm{s}$.

\section{Conclusions}

The conclusion mathematically rigorously proven by Hamed and Frostig [17] was introduced, i.e., zero effect of both bonded and unbonded prestress compression on the natural frequencies of beams as opposed to some research works. It is pointed out that there does exist EP effect on the natural frequencies of beams unlike the zero effect of both bonded and unbonded prestress compression. Miyamoto et al.'s [18] and Shi et al.'s [19] analytical solutions for externally prestressed simply supported and continuous beams were introduced and discussed. The energy method considering the second-order effects was adopted to obtain more accurate solution. The continuous beam frequency results of the energy method in this study, Shi et al.'s [19] analytical solution, and FEM were compared. The results obtained from this study are summarized below:

There exist 4 problems in Miyamoto et al.'s [18] and Shi et al.'s [19] analytical solutions: 2 problems in the proportional assumption that the increment of tendon tension is proportional to the vibration displacement at midspan of beam; 1 problem in the equivalent-area principle that $\Delta M_{\mathrm{P}}$ is simplified into uniform $\Delta M_{\mathrm{PE}} ; 1$ problem in the treatment of EP as $N$.

The influence coefficient of EP, i.e., EP softening effect, mainly depends on the number of contact points and slightly depends on tendon layout. Without deviator, the influence coefficient is equal to 1 , and EP effect is the same as $N$ effect. As the number of contact points increases from minimum value 3 to a large number in continuous beam, the deformation of external tendon is gradually close to unbonded tendon, the loss of external tendon eccentricity reduces, and the influence coefficient decreases from 1 to almost 0 , which is consistent with the conclusion pointed out by Hamed [17]. The loss of external tendon eccentricity results in EP softening effect.
For the beam without deviator, EP effect is the same as $N$ effect, and it may not be negligible. For the beam with one or more deviators, EP effect is much less than $N$ effect and is negligible. As one or more deviators always exist along the continuous beam span, therefore EP effect on frequency is negligible in continuous beam.

The effect of tendon layout linear transformation on the frequency and influence coefficient is negligible.

The first-order tendon deformation is not caused by all antisymmetric modes but is caused mainly by the first symmetric mode, and slightly by other symmetric modes. Therefore, the influence of external tendon on the first symmetric frequency $\left(\omega_{2}\right)$ is obvious; the influence on other frequencies is negligible. As the eccentricity and area of tendon increase, the tendon improves the beam stiffness, and the first symmetric frequency $\left(\omega_{2}\right)$ increases, but other frequencies almost remain unchanged.

\section{Data Availability}

The theoretical investigation was conducted, so the calculation data used to support the findings of this study are included within the article.

\section{Conflicts of Interest}

The author declares no conflicts of interest.

\section{Acknowledgments}

The financial support from National Natural Science Foundation of China (51878302 and 52078225) and Scientific and Technological Planning Guiding Project of Fujian Province (2020Y0087) is greatly appreciated. 


\section{References}

[1] T. Lou, S. M. R. Lopes, and A. V. Lopes, "Effect of linear transformation on nonlinear behavior of continuous prestressed beams with external FRP cables," Engineering Structures, vol. 147, pp. 410-424, 2017.

[2] T. Lou and T. L. Karavasilis, "Numerical assessment of the nonlinear behavior of continuous prestressed steel-concrete composite beams," Engineering Structures, vol. 190, pp. 116$127,2019$.

[3] B. A.-S. Anwar, G. S. Ibrahim, and S. S. Mohamed, "Effect of geometrical properties on strength of externally prestressed steel-concrete composite beams," Proceedings of the Institution of Civil Engineers-Structures and Buildings, vol. 173, no. 1, pp. 42-62, 2020.

[4] D.-P. Fang, "Friction element for non-linear analysis of friction effect in externally prestressed beams," Proceedings of the Institution of Civil Engineers-Structures and Buildings, vol. 173, no. 3, pp. 198-209, 2020.

[5] M. Saiidi, B. Douglas, and S. Feng, "Prestress force effect on vibration frequency of concrete bridges," Journal of Structural Engineering, vol. 120, no. 7, pp. 2233-2241, 1994.

[6] A. Dall'asta and L. Dezi, "Prestress force effect on vibration frequency of concrete bridge-discussion," Journal of Structural Engineering, vol. 122, no. 4, p. 458, 1996.

[7] G. Deák, "Discussion of "prestress force effect on vibration frequency of concrete bridges", Journal of Structural Engineering, vol. 122, no. 4, pp. 458-459, 1996.

[8] S. K. Jain and S. C. Goel, "Discussion of "prestress force effect on vibration frequency of concrete bridges", Journal of Structural Engineering, vol. 122, no. 4, pp. 459-460, 1996.

[9] O. R. Jaiswal, "Effect of prestressing on the first flexural natural frequency of beams," Structural Engineering and Mechanics, vol. 28, no. 5, pp. 515-524, 2008.

[10] K. Kanaka and G. Venkateswara, "Free vibration behavior of prestressed beams," Journal of Structural Engineering, vol. 121, no. 7, pp. 433-437, 1986.

[11] T. H. T. Chan and T. H. Yung, "A theoretical study of force identification using prestressed concrete bridges," Engineering Structures, vol. 22, no. 11, pp. 1529-1537, 2000.

[12] A. Dall'asta and G. Leoni, "Vibration of beams prestressed by internal frictionless cables," Journal of Sound and Vibration, vol. 222, no. 1, pp. 1-18, 1999.

[13] A. D. Kerr, "On the dynamic response of a prestressed beam," Journal of Sound and Vibration, vol. 49, no. 4, pp. 569-573, 1976.

[14] M. Simsek and T. Kocaturk, "Dynamic analysis of eccentrically prestressed damped beam under moving harmonic force using higher order shear deformation theory," Journal of Structural Engineering, vol. 133, no. 12, pp. 1733-1741, 2007.

[15] Z.-C. Wang and W.-X. Ren, "Dynamic analysis of prestressed concrete box-girder bridges by using the beam segment finite element method," International Journal of Structural Stability and Dynamics, vol. 11, no. 2, pp. 379-399, 2011.

[16] J.-Q. Jiang and G.-R. Ye, "Dynamics of a prestressed Timoshenko beam subject to arbitrary external load," Journal of Zhejiang University-Science A, vol. 11, no. 11, pp. 898-907, 2010.

[17] O. Hamed and Y. Frostig, "Natural frequencies of bonded and unbonded prestressed beams-prestress force effects," Journal of Sound and Vibration, vol. 295, no. 1-2, pp. 28-39, 2006.

[18] A. Miyamoto, K. Tei, H. Nakamura, and J. W. Bull, "Behavior of prestressed beam strengthened with external tendons,"
Journal of Structural Engineering, vol. 126, no. 9, pp. 10331044, 2000.

[19] L. Shi, H. He, and W. Yan, "Prestress force identification for externally prestressed concrete beam based on frequency equation and measured frequencies," Mathematical Problems in Engineering, vol. 2014, Article ID 840937, 13 pages, 2014.

[20] M. Simsek and T. Kocaturk, "Nonlinear dynamic analysis of an eccentrically prestressed damped beam under a concentrated moving harmonic load," Journal of Sound and Vibration, vol. 320, no. 1-2, pp. 235-353, 2009.

[21] W. Ji, L. Deng, and P. S. Lin, "Study of vertical bending vibration behavior of continuous prestressed concrete box girders with corrugated steel webs," Advances in Structural Engineering, vol. 19, no. 6, pp. 953-965, 2016.

[22] D.-P. Fang, "Second order effects of external prestress on frequencies of simply supported beam by energy method," Structural Engineering and Mechanics, vol. 52, no. 4, pp. 687-699, 2014. 